\title{
Surfactant-driven escape from endpinching during contraction of nearly inviscid filaments
}

\author{
Pritish M. Kamat ${ }^{1} \dagger$, Brayden W. Wagoner ${ }^{1}$, Alfonso A. \\ Castrejón-Pita ${ }^{2}$, José R. Castrejón-Pita ${ }^{3}$, Christopher R. Anthony ${ }^{1} \ddagger$, \\ and Osman A. Basaran ${ }^{1}$ | \\ ${ }^{1}$ Davidson School of Chemical Engineering, Purdue University, West Lafayette, IN 47907, USA \\ ${ }^{2}$ Department of Engineering Science, University of Oxford, Parks Road, Oxford OX1 3PJ, UK \\ ${ }^{3}$ School of Engineering and Material Science, Queen Mary University of London, Mile End \\ Road, London E1 4NS, UK
}

(Received $\mathrm{xx}$; revised $\mathrm{xx}$; accepted $\mathrm{xx}$ )

Highly stretched liquid drops, or filaments, surrounded by a gas are routinely encountered in nature and industry. Such filaments can exhibit complex and unexpected dynamics as they contract under the action of surface tension. Instead of simply retracting to a sphere of the same volume, low-viscosity filaments exceeding a critical aspect ratio undergo localized pinch-off at their two ends resulting in a sequence of daughter droplets - a phenomenon called endpinching - which is an archetype breakup mode that is distinct from the classical Rayleigh-Plateau instability seen in jet breakup. It has been shown that endpinching can be precluded in filaments of intermediate viscosity, with the so-called escape from endpinching being understood heretofore only qualitatively as being caused by a viscous mechanism. Here, we show that a similar escape can also occur in nearly inviscid filaments when surfactants are present at the free surface of a recoiling filament. The fluid dynamics of the escape phenomenon is probed by numerical simulations. The computational results are used to show that the escape is driven by the action of Marangoni stress. Despite the apparently distinct physical origins of escape in moderately viscous surfactant-free filaments and that in nearly inviscid but surfactant-covered filaments, it is demonstrated that the genesis of all escape events can be attributed to a single cause - the generation of vorticity at curved interfaces. By analyzing vorticity dynamics and the balance of vorticity in recoiling filaments, the manner in which surface tension gradients and concomitant Marangoni stresses can lead to escape from endpinching is clarified.

Key words: Authors should not enter keywords on the manuscript, as these must be chosen by the author during the online submission process and will then be added during the typesetting process (see http://journals.cambridge.org/data/relatedlink/jfmkeywords.pdf for the full list)

$\dagger$ Present address: Dow, Inc., Lake Jackson, TX 77566, USA

$\ddagger$ Present address: Convergent Science, Inc., Madison, WI 53719, USA

ๆ Email address for correspondence: obasaran@purdue.edu 


\section{Introduction}

A liquid filament or ligament is an elongated drop that consists of a slender central section that is capped off by two bulbous ends. Such filaments occur widely in both industrial processes and nature. Well known situations in which they arise include dropon-demand (DOD) ink jet printing (Basaran et al. 2013; Castrejón-Pita et al. 2013) and DOD-based applications in diverse fields (Yeo et al. 2004; Derby 2010) where both the primary/main drops and the satellite droplets resemble filaments, and when satellite droplets form while liquid drips at a low flow rate from a nozzle during particle production and surface tension measurement (Basaran 2002; Zhang et al. 1994). Filaments are also routinely formed when liquid is sprayed from nozzles in crop spraying and atomization coating applications or in naturally occurring fountains and sprays (Eggers 2005; Eggers \& Villermaux 2008). In many of these situations, whether the filament contracts to a single spherical droplet or breaks into many smaller droplets - satellites or fines - is critically important from a performance standpoint.

While filaments come in a variety of configurations (Notz \& Basaran 2004; Notz et al. 2001; Planchette et al. 2019), the most commonly studied type is a so-called symmetric filament whose initial configuration is a cylinder of radius $R$ that is terminated at its two ends by two identical hemispherical caps each of radius $R$ and whose length equals $2 \tilde{L}_{0}$, and the fluid within which is quiescent. In many situations and in the present paper, the fluid exterior to the filament is a passive gas that exerts a constant pressure on the filament. Although liquids used in applications can be simple or complex, the most oft-studied filaments are incompressible Newtonian fluids of constant density $\rho$, viscosity $\mu$, and surface tension $\tilde{\sigma}_{p}$. Because of surface tension, such filaments contract and their final fates, i.e. whether they undergo breakup or no breakup, depend solely on two dimensionless groups: the Ohnesorge number $O h=\mu /\left(\rho R \tilde{\sigma}_{p}\right)^{1 / 2}$ and the initial aspect ratio $L_{0}=\tilde{L}_{0} / R$. The contraction dynamics of such filaments has been studied extensively over the past two decades (Schulkes 1996; Notz \& Basaran 2004; CastrejónPita et al. 2012; Driessen et al. 2013; Wang et al. 2019), culminating with a recent comprehensive study of the dynamics that resulted in the construction of a phase diagram in $O h-L_{0}$ space spanning nearly four orders of magnitude variation in both parameters (Anthony et al. 2019). The goal of this paper is to report novel dynamics that arises when the interface of a nearly inviscid $\left(O h \approx 10^{-3}\right)$ filament is covered with surfactant.

As first shown by Notz \& Basaran (2004) for nearly inviscid filaments, there exists a critical aspect ratio $L_{0, c}$ such that filaments of $L_{0}<L_{0, c}$ do not break while filaments of $L_{0} \geqslant L_{0, c}$ break up. Long, slender filaments of $L_{0} \geqslant L_{0, c}$ that do undergo breakup do so by the endpinching mechanism initially discovered by Stone et al. (1986) who were studying a physical problem that had considerable differences compared to the one being studied here. Although Leal and coworkers (Stone et al. 1986; Stone \& Leal 1989b,a) used an apparatus similar to Taylor's four-roll mill to study the breakup of viscous liquid drops in a dynamically active but less viscous external liquid under Stokes/creeping flow conditions, i.e. under conditions of no inertia or at zero Reynolds number in both the filament and the surrounding fluid, the mechanism of breakup in their studies was virtually identical to that observed by Notz \& Basaran (2004) in whose study inertia was dominant compared to viscous force in the filament and the surrounding fluid was a passive gas. Leal and coworkers showed that stretched drops, when allowed to contract or retract, undergo repeated localized pinch-off near the ends of the filament or undergo endpinching, leaving in the aftermath a train of smaller spherical droplets. While the physics of the contraction of nearly inviscid Newtonian filaments is now fully characterized (Anthony et al. 2019), in many of the aforementioned applications 
surfactants, or surface-active agents which lower surface tension, may be present at the interface by either design or accident. However, despite the existence of a large body of work describing the effect of surfactants in various free surface flows in general and thread breakup in particular (Ambravaneswaran \& Basaran 1999; Liao et al. 2006b; Timmermans \& Lister 2002; McGough \& Basaran 2006; Kamat et al. 2018; MartínezCalvo et al. 2020), investigations on the effects of surfactants on the contraction of filaments surrounded by a passive gas at finite Reynolds number are sorely lacking. Remedying this situation is a goal of this work.

To illustrate the novel physical phenomenon that is of interest, a quick overview will be given of experiments that will be described in greater detail in a future publication (Kamat et al. 2020). Motivated by earlier experimental work by Hoepffner \& Paré (2013) who studied filaments with clean interfaces, in our experiments we fill a fixed vertical tube with liquid and then send the liquid into free fall by gravity, as depicted in Figure 1(a). As the column of liquid exits the tube, a liquid filament forms and resembles one half or end of a free symmetric filament. To quantify the dynamics of pinch-off, the filament's minimum radius $\tilde{R}_{\text {min }}$ is tracked as a function of time $\tilde{t}$. Figure 1 (b) shows the variation of the filament's normalized minimum radius with normalized time for three filaments. For a Newtonian fluid of $O h=0.0017$, the filament pinches off by endpinching and $R_{\text {min }}$ decreases monotonically with time until breakup. Surprisingly, when a surfactant (Sodium dodecyl sulfate, SDS, at a concentration of $3.92 \mathrm{mM}$ ) is added to this Newtonian filament, the dynamics initially follows that of a surfactant-free filament but suddenly changes course such that pinch-off is avoided. This escape from endpinching is well known for Newtonian filaments of larger viscosity, but a comparison of the dynamics of the surfactant-covered filament with a slightly more viscous filament without surfactant of $O h=0.0023$ reveals stark differences between the two cases.

In this work, we use numerical simulations to analyze the effect of insoluble surfactants on the dynamics of contraction of a filament of a Newtonian liquid which would undergo endpinching if its surface were clean or devoid of surfactant. To orient our study, we take a well characterized Newtonian filament of $O h=0.001$ and $L_{0}=15$, and investigate the dynamical variations that take place when insoluble surfactants are deposited on the filament's surface.

It is important to note that SDS used in the aforementioned experiments is soluble in glycerol-water (GW) mixtures. However, its solubility does not affect the surface tension or its gradient along the filament interface during the retraction and pinch-off process because the time scale of the flow is a few milliseconds while the characteristic time for surfactant adsorption-desorption from the interface is hundreds of milliseconds. Therefore, during filament contraction and pinch-off, the surfactant effectively behaves like a purely insoluble surfactant that resides solely on the interface. This fact has been well-demonstrated in recent works studying droplet pinch-off (Roché et al. 2009; Kamat et al. 2018) where numerical simulations considering insoluble surfactants and experiments using SDS in GW mixtures have been shown to be in excellent accord with one another. Hence, the use of the surfactant insolubility assumption is well justified in the simulations.

The rest of the paper is organized as follows. The problem statement and the governing equations are presented in $\S 2$. The numerical method used to solve the governing equations is summarized in $\S 3$. We then report in the following four sections results and discussion of the various aspects of the dynamics as follows. In the next two sections, by taking advantage of simulation results, first we peer into the flows that lead to endpinching when surfactants are absent in $\S 4$, and thereafter in $\S 5$ demonstrate escape from endpinching when surfactants are added. Details of surfactant-driven escape are 

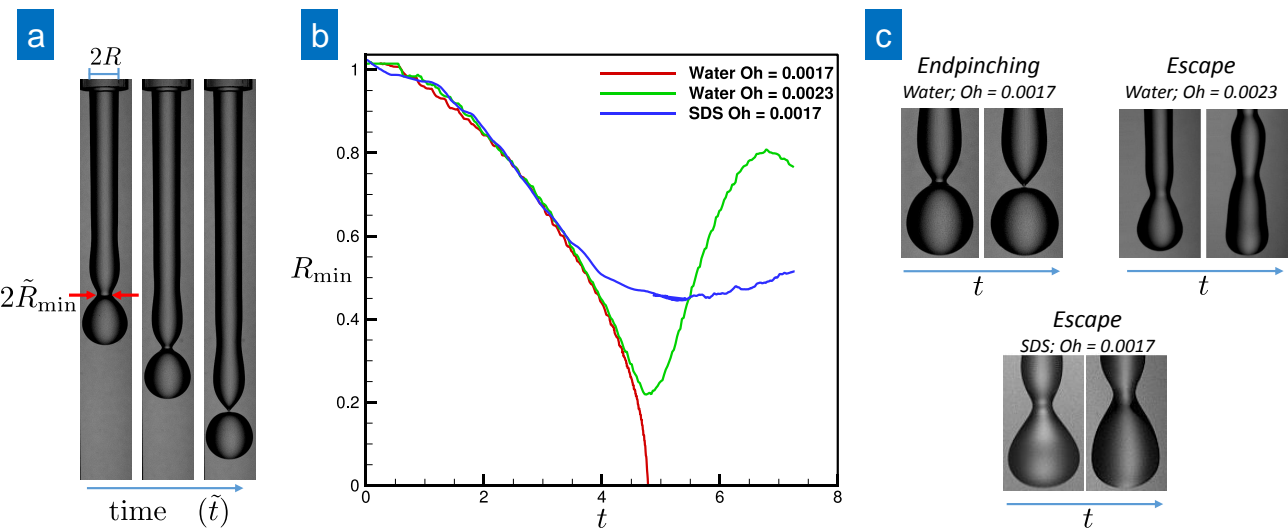

Figure 1. Experimental results on endpinching and escape from endpinching. (a) Images showing the generation or formation of a filament from a nozzle and the evolution in time $\tilde{t}$ of the filament's shape. In the image showing the filament at the earliest time, twice the radius of the filament $2 R$ as it exits from the nozzle and twice the minimum radius of the filament's neck, $2 \tilde{R}_{\text {min }}$, are also identified. (b) Variation with normalized time $t \equiv \tilde{t} / \sqrt{\rho R^{3} / \tilde{\sigma}_{p}}$ of the normalized minimum filament radius $R_{\min } \equiv \tilde{R}_{\min } / R$ from three experiments. Red curve: Water, $O h=0.0017$. Green curve: Water, $O h=0.0023$. Blue curve: Water plus SDS (labeled as "SDS"), $O h=0.0017$. As also shown by the images depicted in the next part of the figure, the filament of water of $O h=0.0017$ endpinches whereas the other two filaments escape from endpinching. (c) Sets of images showing the filaments at two instants in time. Endpinching: water filament of $\mathrm{Oh}=0.0017$ prior to (left) and at the incipience of (right) endpinching. Escape (Water): water filament of $O h=0.0023$ appearing on the cusp of breaking (left) and then shortly after it has escaped endpinching (right). Escape (SDS): surfactant-covered filament of $O h=0.017$ appearing on the verge of endpinching (left) and then after it has undergone surfactant-driven escape from endpinching (right). In (a) and (c), the arrows indicate the direction in which time, $\tilde{t}$ or $t$, is increasing.

provided in $\S 6$ where we observe that surfactants can abet the escape from endpinching through a mechanism different than the boundary-layer proposal of Hoepffner \& Paré (2013), and show conclusively that this process is primarily driven by Marangoni stresses acting in the vicinity of the about-to-pinch neck of a retracting filament. In $\S 7$, an analysis is presented of the key role of vorticity in all escape phenomena. $\S 8$ provides concluding remarks and a short summary of future work that can be undertaken for advancing this field.

\section{Problem Statement and Mathematical Formulation}

The system, shown in figure 2 , is an ideal filament which, as discussed in section 1 , is an axisymmetric cylinder of radius $R$ and finite length $2 \tilde{L}_{0}$ with hemispherical caps at its two ends. The filament is comprised of an isothermal, incompressible Newtonian liquid (or, solvent) with constant density $\rho$ and viscosity $\mu$, and is surrounded by a dynamically passive gas that exerts a constant pressure, which is taken to be the pressure datum, on the filament. The surface tension of the L-G interface separating a filament of pure liquid from the exterior passive gas is $\tilde{\sigma}_{p}$. The fluid within the filament is initially quiescent or at rest, viz. the fluid velocity $\tilde{\mathbf{v}}=\mathbf{0}$ at time $\tilde{t}=0$. The filament's surface is covered with an insoluble, non-volatile surfactant. At $\tilde{t}=0$, the initial concentration of surfactant is uniform and given by $\tilde{\Gamma}_{0}$, and the resultant initial surface tension distribution $\tilde{\sigma}_{0}$ is also uniform and is such that $\tilde{\sigma}_{0} \leqslant \tilde{\sigma}_{p}$. We employ a cylindrical coordinate system $(\tilde{r}, \theta, \tilde{z})$ 

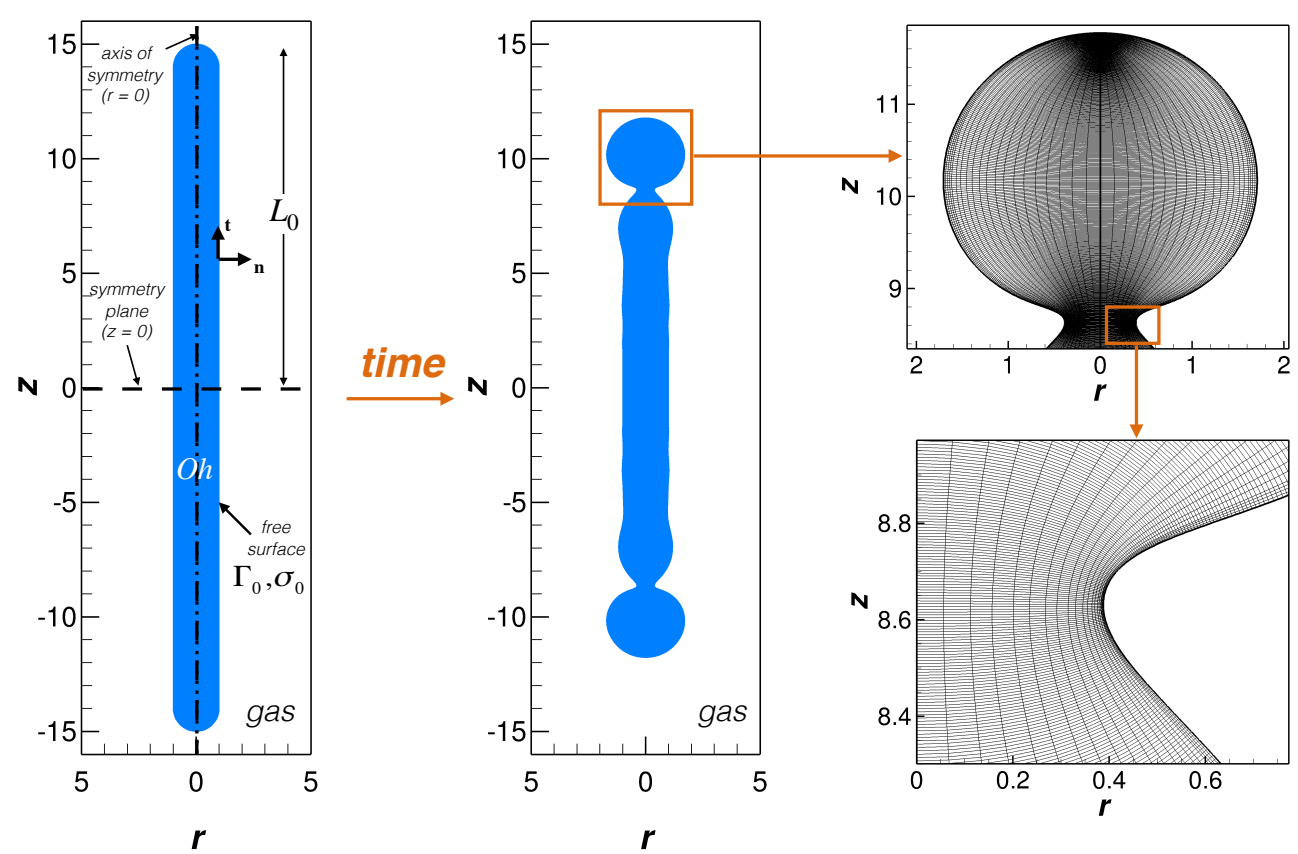

FiguRE 2. A contracting surfactant-laden filament: definition sketch and computational mesh/grid used in simulations. Left: The initial state of the filament. Center: The filament after it has contracted for some time. Right: A blow-up or zoomed-in view of the computational mesh constructed by elliptic mesh generation in the bulbous end and neck regions (top) and a further zoomed-in view of the mesh in the neck (bottom). In parts of this figure (the left-most and center frames) and some others in the rest of the paper, following pioneering works in the field (see, e.g., Stone \& Leal $(1989 b)$ ), cross-sections of entire filaments are shown even though the filaments are axisymmetric and have a plane of symmetry. As explained in the text, the problem domain, however, consists of one-quarter of a filament's cross-section in the $(r, z)$-plane.

where $\tilde{r}$ and $\tilde{z}$ are the radial and axial coordinates measured from the center of mass of the filament, and $\theta$ is the azimuthal angle. Due to the axisymmetry of the problem, the dynamics are independent of $\theta$ and the problem can be effectively reduced to a two-dimensional problem in $\tilde{r}$ and $\tilde{z}$. Moreover, it is taken here that the dynamics is mirror-symmetric about the midplane of the filament $(\tilde{z}=0)$. Thus, the problem domain consists of one quadrant of the $(\tilde{r}, \tilde{z})$ plane, where $\tilde{r}, \tilde{z} \geqslant 0$, that is bounded by the axis of symmetry, the plane of symmetry, and the filament's free surface.

The problem is cast in dimensionless form by normalizing length with $R$, time with the inertial-capillary time scale $t_{c}=\left(\rho R^{3} / \tilde{\sigma}_{p}\right)^{1 / 2}$, stress with $\mu / t_{c}$, the surfactant concentration with its value at maximum packing $\tilde{\Gamma}_{m}$, and surface tension with $\tilde{\sigma}_{p}$. Hereafter, variables without tildes over them will denote the dimensionless counterparts of those with tildes, e.g. $\tilde{t}$ and $t$ stand for dimensional and dimensionless time.

The bulk flow of the viscous liquid within the contracting filament is governed by the incompressible forms of the dimensionless continuity and Navier-Stokes equations

$$
\begin{aligned}
\nabla \cdot \mathbf{v} & =0 \\
\frac{\partial \mathbf{v}}{\partial t}+\mathbf{v} \cdot \nabla \mathbf{v} & =\nabla \cdot \mathbf{T}
\end{aligned}
$$

where $\mathbf{T}=-p \mathbf{I}+O h\left[\nabla \mathbf{v}+(\nabla \mathbf{v})^{T}\right]$ is the total stress tensor for a Newtonian fluid, $\mathbf{I}$ is 
the identity tensor, and $p$ is the pressure. At the L-G interface, the counterparts of the previous two equations are of course the kinematic and traction boundary conditions

$$
\begin{aligned}
& \mathbf{n} \cdot\left(\mathbf{v}-\mathbf{v}_{s}\right)=0 \\
& \mathbf{n} \cdot \mathbf{T}=2 \mathcal{H} \sigma \mathbf{n}+\nabla_{s} \sigma
\end{aligned}
$$

where $\mathbf{n}$ is the outward pointing unit normal, $\mathbf{v}_{s}$ is the instantaneous local velocity of the free surface, $2 \mathcal{H}=-\nabla_{s} \cdot \mathbf{n}$ is twice the local mean curvature of the free surface, and $\nabla_{s}=\nabla-\mathbf{n n} \cdot \nabla$ is the surface gradient operator. The tangential component of the traction vector $\mathbf{n} \cdot \mathbf{T}$ is non-trivial only when there is a gradient of surface tension $\sigma$ at the free surface, and is commonly referred to as the Marangoni stress (Scriven \& Sternling 1960)

$$
\mathbf{n} \cdot \mathbf{T} \cdot \mathbf{t} \equiv T_{n t}=\mathbf{t} \cdot \nabla_{s} \sigma
$$

where $\mathbf{t}$ is the unit tangent to the free surface. The surface tension $\sigma$ of the free surface is related to the local concentration of surfactant $\Gamma$ on the surface by the Szyszkowski equation of state (Rosen 2004; Liao et al. 2006b)

$$
\sigma=1+\beta \ln (1-\Gamma)
$$

where $\beta=\tilde{\Gamma}_{m} R_{g a s} T_{a b s} / \tilde{\sigma}_{p}$ is the dimensionless surfactant strength parameter. Here $R_{\text {gas }}=8.314 \mathrm{~J} / \mathrm{mol}-\mathrm{K}$ is the universal gas constant and $T_{a b s}$ is the absolute temperature of the isothermal system. Additionally, the bulk flows can convect and redistribute the surfactant species on the free surface. The surfactant concentration is governed by the surface convection-diffusion equation (Stone 1990) which, separating the fundamental transport mechanisms - convection $\dot{\Gamma}_{c o n}$, normal dilatation $\dot{\Gamma}_{d i l}$, tangential stretching $\dot{\Gamma}_{s t r}$, and diffusion $\dot{\Gamma}_{d i f}$-is given by

$$
\frac{\partial \Gamma}{\partial t}+\underbrace{\mathbf{v} \cdot \nabla_{s} \Gamma}_{\text {Convection }}+\underbrace{\Gamma v_{n}\left(\nabla_{s} \cdot \mathbf{n}\right)}_{\text {Normal dilatation }}+\underbrace{\Gamma\left(\nabla_{s} \cdot v_{t} \mathbf{t}\right)}_{\text {Tangential stretching }}-\underbrace{\frac{1}{P e} \nabla_{s}^{2} \Gamma}_{\text {Diffusion }}=0
$$

where $v_{n}=\mathbf{n} \cdot \mathbf{v}$ and $v_{t}=\mathbf{t} \cdot \mathbf{v}$ are the components of the velocity normal and tangential to the free surface, $P e=R^{2} /\left(t_{c} D_{s}\right)=D_{s}^{-1}\left(R \tilde{\sigma}_{p} / \rho\right)^{1 / 2}$ is the surface Péclet number measuring the relative importance of convection to diffusion, and $D_{s}$ is the surface diffusivity of the surfactant at the interface.

The system is axisymmetric about the $z$-axis, or $r=0$, and symmetric about the plane $z=0$; along these boundaries, the following boundary conditions are imposed on the flow field:

$$
\mathbf{n} \cdot \mathbf{T} \cdot \mathbf{t}=0 \quad \text { and } \quad \mathbf{n} \cdot \mathbf{v}=0 .
$$

The filament shape and surfactant concentration also obey axisymmetry and symmetry boundary conditions at $r=0$ and $z=0$ where the free surface profile intersects the $z$ axis and the plane of symmetry, respectively. For the filament profile, the two symmetry conditions are imposed as $\mathbf{t} \cdot \mathbf{e}_{z}=0$ and $\mathbf{t} \cdot \mathbf{e}_{r}=0$, where $\mathbf{e}_{z}$ and $\mathbf{e}_{r}$ are unit vectors in the axial and radial directions. For the surfactant concentration, the symmetry conditions are imposed by setting $\mathbf{t} \cdot \nabla_{s} \Gamma=0$ at both locations.

\section{Numerical Method}

The simulations rely on an algorithm that is based on the finite element method (Strang \& Fix 1973) and a sharp interface representation of the free surface of the filament. More specifically, equations (2.1) to (2.8) are solved simultaneously as an 


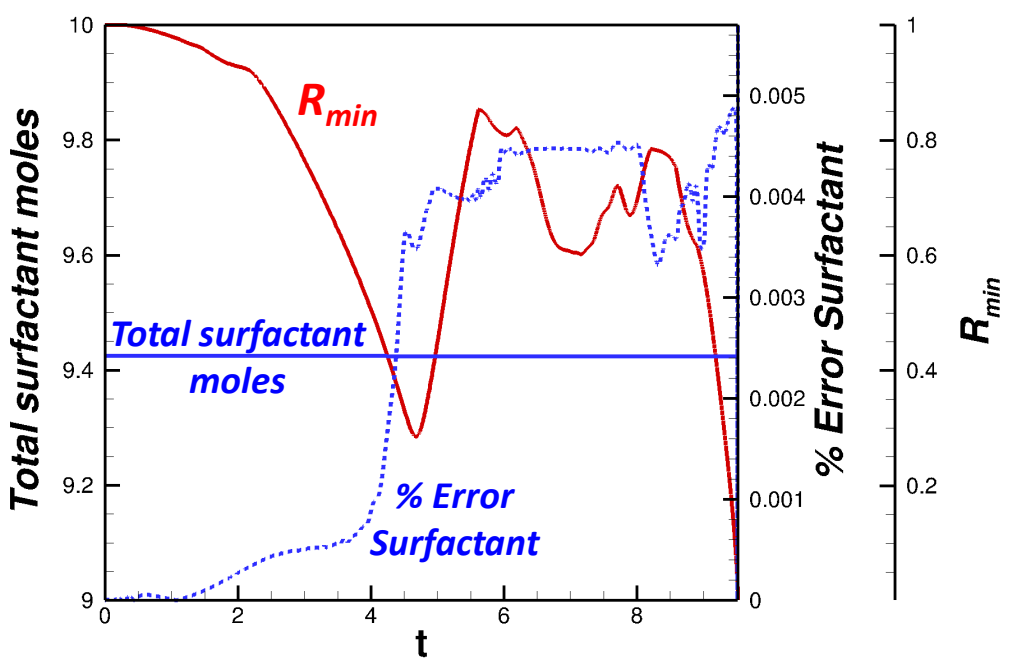

Figure 3. Variation of total moles of surfactant (solid blue line, "Total surfactant moles"), percent change in the total amount of sufactant (dotted blue curve, "\% Error Surfactant"), and minimum neck radius $R_{\text {min }}$ (solid red curve) with time $t$. The total amount of surfactant is that for one half of the filament, the initial value of which equals $(0.1)(30 \pi)$. It should be noted that different axes are used for the three dependent variables. Here, $O h=0.001, L_{0}=15, \Gamma_{0}=0.1$, $\beta=0.05$, and $P e=10$.

initial-boundary-value problem by a fully implicit, method of lines (MOL), arbitrary LagrangianEulerian (ALE) algorithm using the Galerkin finite element method (G/FEM) with elliptic mesh generation (Christodoulou \& Scriven 1992) for spatial discretization and a finite difference predictor-corrector scheme for time integration with adaptive time step-size control (Gresho et al. 1979).

The code was benchmarked against the simulations of Notz \& Basaran (2004) on contraction of surfactant-free filaments and those of Liao et al. (2006b) on pinch-off of surfactant-covered liquid bridges. Additionally, the code conserved volume and surfactant moles with a relative error of less than $0.1 \%$ (cf. figure 3 ). Details of the numerical technique, including the implementation of the elliptic mesh generation algorithm, can be found in Notz \& Basaran (2004) and Liao et al. (2006b).

\section{Endpinching of Surfactant-free Filaments}

Figure 4(a) shows the evolution in time of the shape of a filament of $L_{0}=15$ and $O h=0.001$. On account of the initial shape of the filament (leftmost frame), a capillary pressure gradient exists at $t=0$ between the hemispherical tip where the pressure equals two and the cylindrical body of the filament where the pressure equals unity. This pressure difference causes liquid to flow from the hemispherical tip into the cylinder. At low $O h$, the compressional stress produced by the flow does not diffuse effectively into the cylindrical portion of the filament and within which the fluid remains stationary at early times. Therefore, when fluid flowing from the tip encounters viscous resistance on account of the existence of the stationary body fluid in the cylinder, its motion is instantly arrested and a stagnation zone forms close to the tip. As the tip recedes, fluid continues to accumulate at this stagnation zone, causing the free-surface to bulge - a key initial step in the dynamical 
a
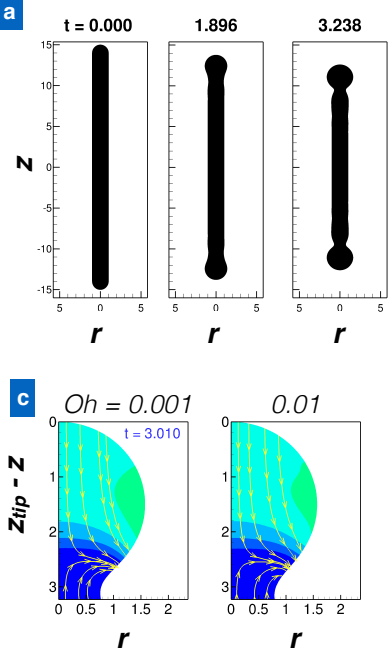

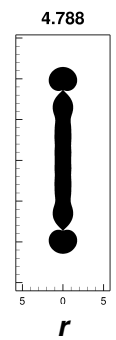

b
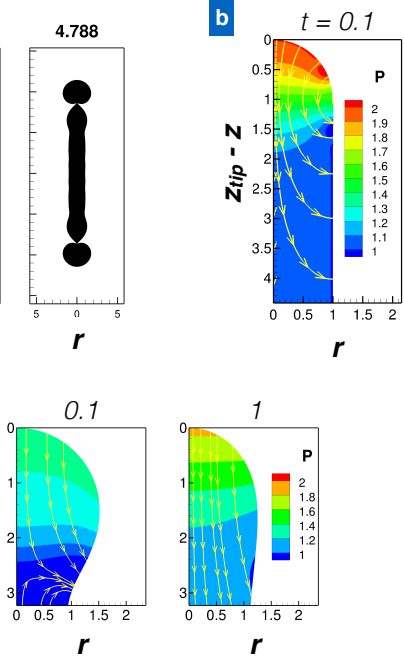
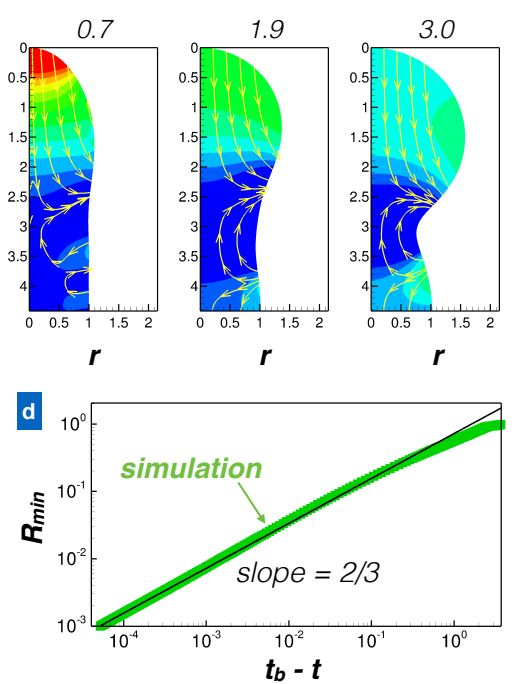

FiguRE 4. Endpinching and bulb formation in surfactant-free filaments. (a) Time evolution of the shape of a filament of $O h=0.001$ and $L_{0}=15$ undergoing endpinching. (b) Bulb formation process for the filament from part (a). (c) Snapshots at $t=3.01$ of bulged tips of filaments of $O h=0.001,0.0,0.1$ and 1. For all filaments, the initial aspect ratio $L_{0}=15$. (d) Scaling of the computed value of the minimum neck radius $R_{\text {min }}$ (green symbols, simulation) with time from breakup $t_{b}-t$ for the filament of part (a) agrees with that predicted by inertio-capillary scaling theory (black line of slope $=2 / 3$ and intercept $=\ln (0.712)$

). Here, as in (a), $O h=0.001$ and $L_{0}=15$.

response of all endpinching filaments. As the bulb grows, the in-plane curvature of the interface becomes more negative where the bulge connects to the cylindrical section of the filament. At the same time, as shown in Figure 4(b), capillary waves are excited on the surface of the cylindrical thread which get progressively weaker with increasing distance measured from the filament's tip.

Once the bulb is formed and capillary waves are triggered, the dynamics that ensues and eventually results in the thinning and breakup of the filament's neck follows the intermediate and late stages of inertio-capillary pinch-off (Chen \& Steen 1997; Day et al. 1998). During the early stages of the dynamics, the pressure in the neck is small owing to the large negative in-plane curvature at that location and is surrounded on either side by two higher pressure regions - the bulb and the bulged section of the thread. During this period, the thinning of the tread is set by the upward flow in the neck which is driven by the positive thread-side pressure drop, as shown Figure 4(b) at $t=1.9$ and 3.0. Also, during this period, as the fluid axially accelerates in the neck, $\partial v / \partial z>0$. From either the general equation of conservation of mass (2.1) or slender-jet theory (Eggers \& Dupont 1994), the radial velocity to the leading order is given by $u \approx-(r / 2) \partial v / \partial z$. Hence, both the fluid and the interface move radially inward such that at the filament's surface $u \approx d R_{\text {min }} / d t<0$. Plainly, the thinning process is self-aggrandizing: as the neck thins, the pressure gradient grows, thus resulting in stronger upward flow in the neck, which in turn further accelerates the thinning of the neck. As an aside, it is instructive to note that the extent of bulb and neck formation and the accelerated thinning of the neck, and the propensity to endpinch become diminished and eventually disappear as $O h$ increases, as shown in figure 4(c) for filaments for which the Ohnesorge number has been increased by a decade in succession over the range 0.001 to 1 . Returning back to the case of the filament 

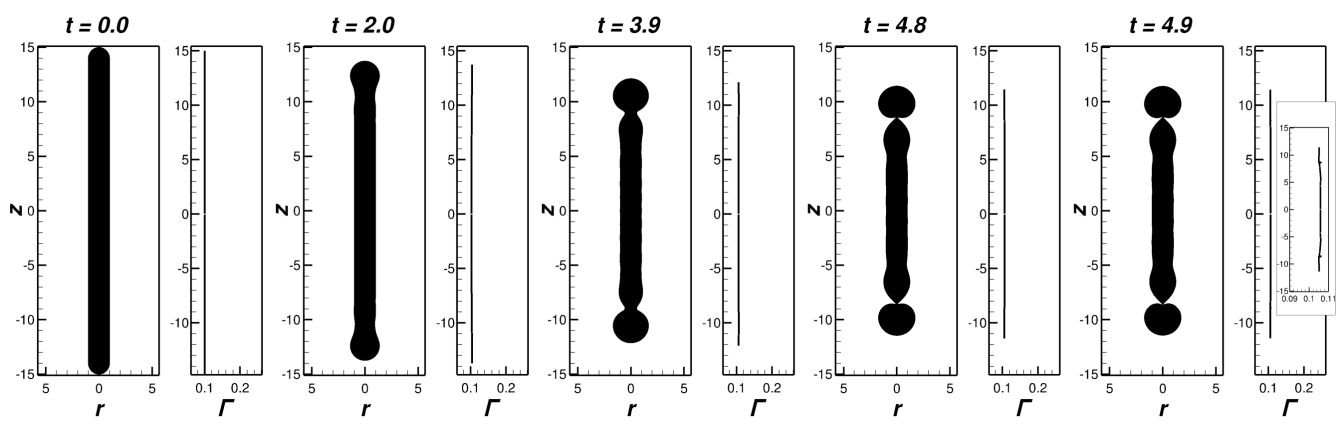

FiguRE 5. Endpinching of a surfactant-covered filament. Time evolution of the computed shape and surfactant concentration at the interface of a contracting filament of $O h=0.001, L_{0}=15$, $\Gamma=0.1, \beta=0.3$, and $P e=0.01$. In this figure and the next, at each instant in time, the filament shape is shown on the left and the surfactant concentration $\Gamma$ on the right. At the latest time $t=4.9$, a zoomed-in view of the surfactant concentration profile is shown as an inset where the range of axis values of $\Gamma$ varies between 0.09 and 0.11 .

of $O h=0.001$, Figure $4(\mathrm{~d})$ shows the radial scaling predicted from the simulations which depicts the variation of $R_{\min }$ with time measured from pinch-off, $t_{b}-t$ where $t_{b}$ stands for the breakup time, for a filament undergoing endpinching. The computed results and the value of the radial scaling exponent and pre-factor of 0.712 determined from the simulations are seen to be in excellent accord with inertio-capillary breakup theory which predicts a power-law exponent of 2/3 (Chen \& Steen 1997; Day et al. 1998) and a pre-factor of $\approx 0.7$ (Eggers \& Villermaux 2008), and thereby confirm the dominant force balance involved in the dynamics of endpinching. In summary, endpinching is a hybrid process consisting of the initial formation of the bulb, neck, and capillary waves, which then gives way to a dynamics where the neck follows and ultimately succumbs to pinch-off as dictated by inertio-capillary breakup theory.

In the next section, a uniform monolayer of surfactant is added to the filament so that $\Gamma(s, t=0)=\Gamma_{0}$ where $s$ is arclength measured along the $\mathrm{L}-\mathrm{G}$ interface. By focusing almost exclusively on filaments of $O h=0.001$ and $L_{0}=15$, the values of $\beta$ and $P e$ are varied to elucidate the dynamical modifications that take place in filament contraction due to the presence of surfactants at the L-G interface.

\section{Surfactant-driven Escape from Endpinching}

Figure 5 shows the instantaneous shape and surfactant concentration profile along the L-G interface during the retraction of a surfactant-laden filament whose surface is initially covered uniformly with surfactant at a concentration given by $\Gamma_{0}=0.1$, and for which $\beta=0.3$ and $P e=0.01$. Much like its surfactant-free counterpart, this filament endpinches and its breakup shape is virtually identical to that of its surfactant-free counterpart. For the surfactant-covered filament, because $P e$ is small, surfactant diffusion is dominant over surfactant convection which, therefore, results in the surfactant density $\Gamma$ profile remaining virtually perfectly uniform throughout the filament's retraction. The presence of surfactant in this case hence merely serves to uniformly lower the dimensionless surface tension of the filament below unity. As the retraction/endpinching process is driven by surface tension, the contraction process is therefore slower and the breakup time is slightly larger for the surfactant-covered filament compared to its surfactant-free counterpart shown in figure $4(\mathrm{a})$. Next, we increase $P e$ by four orders of magnitude to investigate 

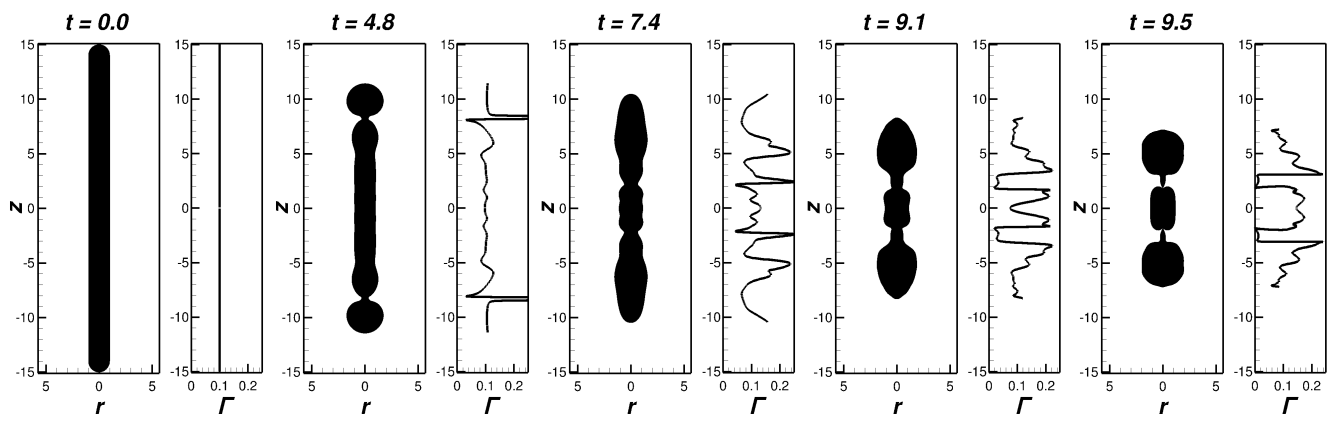

FiguRE 6. Escape from endpinching and eventual breakup of a surfactant-covered filament. Time evolution of the computed shape and surfactant concentration at the interface of a contracting filament of $O h=0.001, L_{0}=15, \Gamma=0.1, \beta=0.05$, and $P e=10$.

whether convection of surfactants has a more profound effect on filament retraction than what has been observed so far.

Figure 6 shows the time evolution of the shape and surfactant concentration for a filament of $\Gamma=0.1, \beta=0.05$, and $P e=10$. The breakup shape in this case $(P e=10)$ looks drastically different compared to the earlier case when $P e=0.01$ : while the filament of $P e=10$ appears to have endpinched, it is markedly shorter at breakup compared to the filament of $P e=0.01$ and the three droplets that result from the breakup of the filament of $P e=10$ are quite similar in size. The filament shape and surfactant concentration profiles at $t=4.8$ in Figure 6 are noteworthy from the following standpoints. First, the surfactant concentration has largely remained unchanged within the blob and also, albeit to a lesser degree, in the cylindrical thread until this instant in time. Second, the strong upward flows in the neck tend to convect surfactant from the thread-side portion of the neck into the bulb, thereby producing a large gradient in $\Gamma$ at the neck. Third, although the dynamics up to this point for the filament of moderate Peclet number $(P e=10)$ is virtually identical to that in an endpinching filament of lower Peclet number $(P e=0.01)$, the similarities between the dynamics in the two cases end after this point in time. Beyond this point, the neck of the filament of $P e=10$ stops thinning and begins to reopen, and large-amplitude capillary waves form in what was earlier the cylindrical section of the filament. The flows accompanying the capillary waves cause the surfactant concentration to become highly non-uniform along the previously cylindrical body of the filament. As the filament contracts further, the local filament radius at the second (as one moves from the filament's tip towards its center) local minimum in the interface shape profile begins to decrease rapidly in time. As the thinning continues and the filament further contracts, the inertio-capillary breakup mechanism kicks-in and the filament undergoes pinch-off at that locale. At pinch-off, two large droplets located at the two ends of the filament are connected to the center satellite droplet by short tail-like structures, as shown by the profile at $t=9.5$ in Figure 6.

It is well-known that surfactant-free filaments of intermediate Ohnesorge numbers, $O h \approx 0.01$, can escape endpinching by a viscous mechanism (Hoepffner \& Paré 2013). It is also known that this mechanism, however, is inoperative in low $O h$ filaments and a surfactant-free filament of $O h=0.001$ of any initial aspect ratio $L_{0} \geqslant 6$ must break by endpinching (Anthony et al. 2019). At first, it seems plausible that endpinching is arrested in the presence of surfactants because the concomitant lowering of surface tension raises the effective $O h$ of the surfactant-laden filament above the value of 0.001 for the surfactant-free filament. As the surface tension in the blob remains nearly uniform and 
equal to its initial value before the escape event, one can compute an effective Ohnesorge number as $O h_{\mathrm{eff}}=\mu /\left(\rho R \tilde{\sigma}_{0}\right)^{1 / 2}=O h / \sqrt{\sigma_{0}}$. However, for $O h=0.001, \beta=0.2$, and $\Gamma_{0}=0.1$, the resulting value of the effective Ohnesorge number $O h_{\mathrm{eff}}=1.03 \times 10^{-3}$. As this value of the effective Ohnesorge number $O h_{\text {eff }}$ is so close to the value of $O h$, surface tension lowering alone cannot possibly be responsible for escape from endpinching. Moreover, if merely lowering surface tension or increasing the effective Ohnesorge number is the cause of the escape, this fact does not explain why the escape process does not occur at small $P e$. When they are all taken together, the previously listed observations imply that Marangoni stress $T_{n t}$ arising from surface tension gradients due to surfactant concentration gradients when $P e \gg 1$, as in Figure 6, may play a critical role in the contraction of surfactant-covered filaments. In the following section, we describe the mechanics of surfactant-driven escape from endpinching and provide insights into the fundamental forces at play.

\section{The Mechanism of Surfactant-driven Escape from Endpinching}

It was discussed in section 4 how endpinching is a cumulative outcome of the localized dynamics of initial bulb and neck formation, which is then followed by the inertio-capillary acceleration of the fluid through the neck from the thread into the bulb. Therefore, when a filament escapes endpinching, one of these processes must be dynamically arrested. We show here that in surfactant-laden filaments, the first of the aforementioned effects, blob and neck formation, proceeds as normal whereas the latter one, inertio-capillary thinning and pinch-off, is strongly influenced by the presence of surfactants. In order to gain insights into how the physics plays out, it is instructive first to examine the manner in which the bulk flows affect the distribution of surfactant at the free surface.

Figure $7(\mathrm{a})$ depicts at an early time $(t=2.1)$ the filament of figure 6 and certain details of the flow within it and surfactant transport along its interface. Here and throughout figure 7, the axial distance from the tip $z_{t i p}-z$ is used as the horizontal axis in lieu of the axial coordinate $z$. The top frame in figure 7 (a) shows the filament in which attention is focused on the region near the tip. Also shown there are the instantaneous streamlines and contours of the axial velocity within the filament. At this instant in time, the retraction process has been under way for some time and bulb formation at the tips has already been initiated. Under the capillary pressure gradient, the fluid flows from the filament's tip toward the filament's center at the Taylor-Culick (Taylor 1959; Culick 1960) (or capillary) velocity scale so that $v=-1$ in the large region adjacent to the filament's tip. Hindered by the viscous resistance that it feels as the fluid moves downstream, the flow then sharply turns radially outward and begins accumulating underneath the free surface a small distance $z_{t i p}-z=2.3 \sim \mathcal{O}(1)$ from the tip. The second and third frames from the top in figure 7 (a) show the variation of surfactant concentration $\Gamma$ and the instantaneous rate of change of concentration $\dot{\Gamma}$ as a function of $z_{t i p}-z$ at the free surface. The bottom frame of figure $7(\mathrm{a})$ shows the detailed breakdown of the processes that contribute to the net rate of change of $\Gamma$-namely, those due to convection $\dot{\Gamma}_{\text {conv }}$ (solid line), normal dilatation of the free surface $\dot{\Gamma}_{d i l}$ (dashed line), tangential stretching of the free surface $\dot{\Gamma}_{\text {str }}$ (dashed-dotted line), and diffusion $\dot{\Gamma}_{\text {diff }}$ (dotted line). Since surfactant is evacuated from the tip, $\dot{\Gamma}<0$ there due nearly entirely to convection, and on account of the fact that dilatation and stretching caused by the bulging of the tip cancel each other out and, moreover, because diffusion is negligible. The surfactant that is transported away from the tip then accumulates near the stagnation zone at the free surface near the nascent neck. There, $\dot{\Gamma}>0$ primarily due to the tangential constriction $\dot{\Gamma}_{s t r}>0$ of the free 

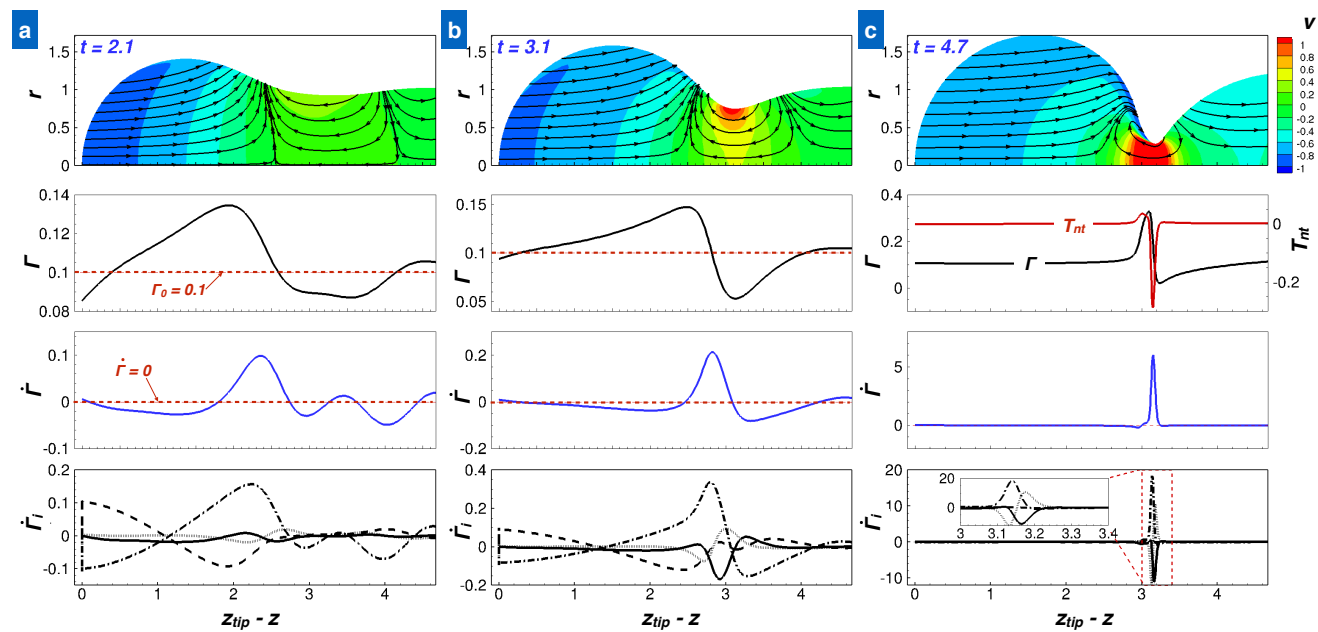

Figure 7. Surfactant redistribution and Marangoni stress generation leading to but before escape from endpinching for the filament of figure 6. Top panels: instantaneous shapes of and streamlines and axial velocity $v$ contours within the filament. Second from top panels: instantaneous surfactant concentration $\Gamma$ and, in one case, Marangoni stress $T_{n t}$, profiles along the filament's free surface. Second from bottom panels: profile of the instantaneous rate of change of surfactant concentration $\dot{\Gamma}$ along the filament. Bottom panels: profiles of the individual contributions $\dot{\Gamma}_{i}$ to the total rate of change $\dot{\Gamma}$ (see text) along the filament. (a) Dynamics at early times: $t=2.1$. The value of the initial surfactant concentration $\Gamma_{0}=0.1$ is stated in the second panel and marked by a dashed straight line both here and at the next instant in time. The location where $\dot{\Gamma}=0$ is shown here and marked by a dashed straight line here and at the next instant in time. (b) Dynamics once the blob has formed: $t=3.1$. (c) Dynamics just before escape: $t=4.7$. In the second panel, the Marangoni stress $T_{n t}$ profile is shown along with the surfactant concentration $\Gamma$ profile.

surface. The variation of $\Gamma$ along the free surface at this stage is small, $\sim \mathcal{O}(0.01)$, as the flow is weak and the amplitude of the surface deformation is small.

Figure 7(b) shows the shape and details of the flow within and surfactant transport along the interface for the same filament as in figure 7 (a) albeit at a later time $(t=3.1)$. Here, the bulge has assumed an almost spherical blob-like shape, a distinct minimum has formed in the neck connecting the blob to the cylinder, and the inertio-capillary flow has been setup in the neck. The endpinching process has thus been initiated. The axial velocity is now larger in the neck, with the flow initiating from the thread-side stagnation zone and terminating in the blob-side stagnation zone. The surfactant concentration $\Gamma$ peaks at the blob-side stagnation zone due to prior accumulation, and drops suddenly and precipitously at the neck. As the velocities are still $\mathcal{O}(1)$ in the neck, convection is relatively weak, and tangential stretching is the dominant mechanism affecting $\dot{\Gamma}$. The slow growth of the blob lends it a small, negative $\dot{\Gamma}$ owing to normal dilatation. The large peak in the $\dot{\Gamma}$ profile occurs near the blob-side stagnation zone due to strong tangential constriction $\left(\dot{\Gamma}_{\text {str }}>0\right)$ at that location, whereas the negative drop in the neck is mainly due to the tangential stretching $\left(\dot{\Gamma}_{s t r}<0\right)$ caused by the flow. The nature of the flow and surfactant transport that has just been described thereby result in the creation of a large surface gradient in $\Gamma$ on the immediate blob-side of the neck. This state of affairs and the magnitude of the surface gradient only exacerbate in time, as made evident by figure $7(\mathrm{c})$ which shows the same filament at yet a later time $(t=4.7)$. While diffusion is active in trying to dampen these effects as $P e$ is in the intermediate range in this case, 

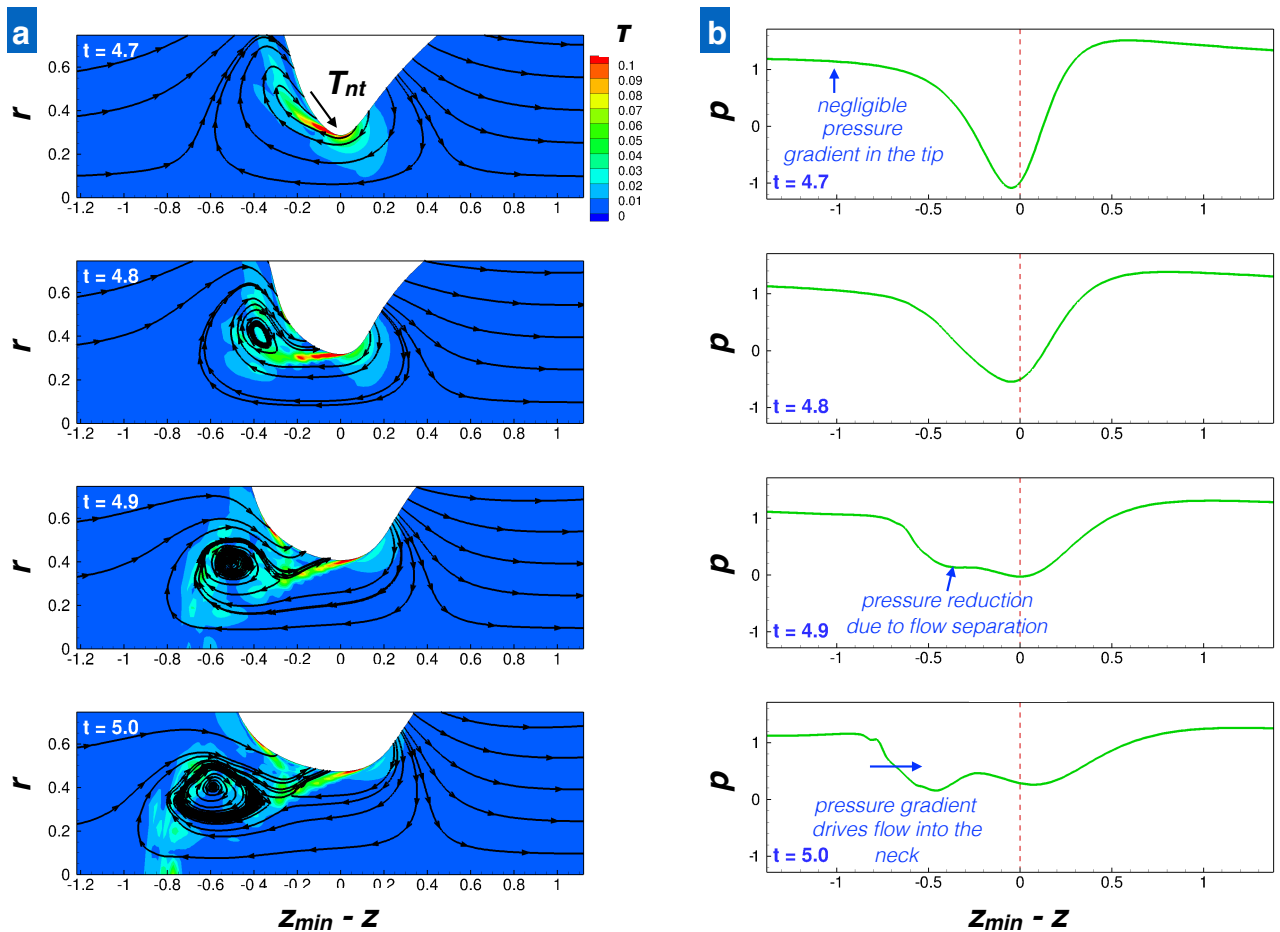

Figure 8. Physics of surfactant-driven escape from endpinching: zoomed-in views of the neck showing flow, stress, and pressure profiles. (a) Top to bottom: evolution in time of the filament profiles near the neck and instantaneous streamlines and stress contours (see text) within the filament. Contour values of the stress $T$ are shown at top right. A large Marangoni stress $T_{n t}$ already exists at the interface at the earliest time, as indicated in the panel at $t=4.7$. As the panels from top to bottom show, the vortex ring is clearly visible at $t=4.8$ and the vortex has detached from the surface at $t=5.0$. (b) Pressure profiles during escape show the "head-loss" that occurs due to flow separation, as predicted by Hoepffner \& Paré (2013).

it is fighting a losing battle and is hence overshadowed by the dominant role played by tangential stretching.

In the second from the top panel of figure $7(\mathrm{c})$, it proves instructive at $t=4.7$ to plot the Marangoni stress profile along with the surfactant concentration profile. The Marangoni stress $T_{n t}$ is given by

$$
T_{n t}=-\frac{\beta}{1-\Gamma}\left(\mathbf{t} \cdot \nabla_{s} \Gamma\right)
$$

The figure makes plain that a large negative spike in the Marangoni stress profile exists just upstream of the neck at this instant in time. Moreover, $\dot{\Gamma}$ is large near the blobside stagnation zone owing predominantly to tangential stretching, which only serves to enhance the magnitude of the negative $T_{n t}$ in this region.

The aforementioned growing Marangoni stress runs counter to the direction of the inertio-capillary flow in the neck. As a result, the free surface acts similar to the moving plate in an axisymmetric driven cavity and is tantamount to a source of shear stress that is opposed to the direction of flow. The top frame of figure 8(a) shows a magnified view of the neck at this time. Here, the contours depict the magnitude of the viscous stress $\tau=O h \dot{\gamma}$, where $\dot{\gamma}$ is twice the second invariant of the rate-of-deformation tensor $\mathbf{D}=\nabla \mathbf{v}+(\nabla \mathbf{v})^{T}$. It can be clearly seen from this figure that the large spike in Marangoni 


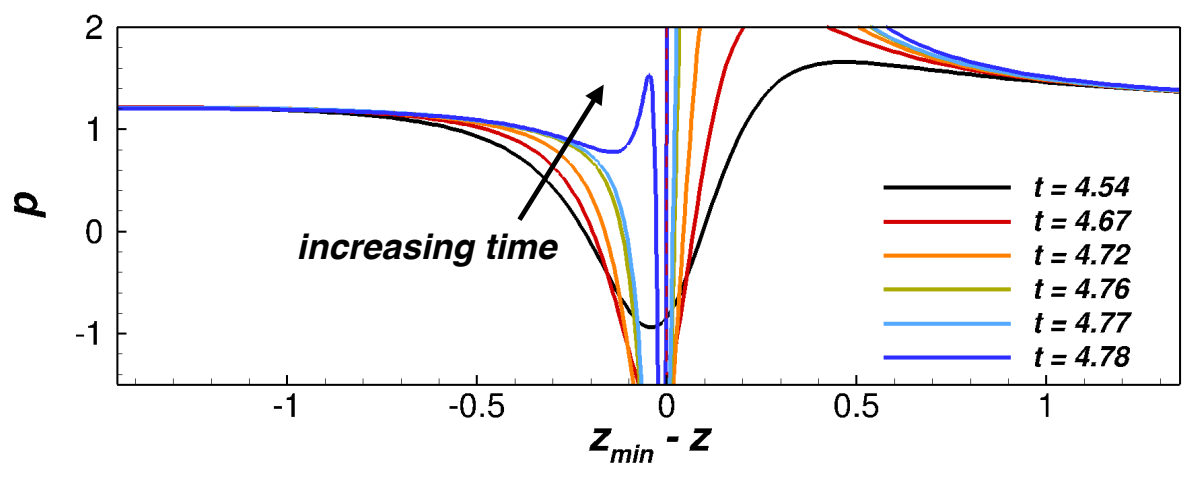

Figure 9. Time evolution of pressure profiles in the blob of a surfactant-free filament undergoing endpinching. Here, $O h=0.001$ and $L_{0}=15$. Notice the absence of the "head-loss", or the secondary pressure minimum in the blob $\left(z_{\min }-z<0\right)$ that is seen in the surfactant-laden case (cf. figure 8b).

stress $T_{n t}$ lends a high value of $\tau$ that is concentrated at the free surface near the neck. This stress causes the fluid on the blob-side of the neck to reverse direction in a thin boundary layer that is adjacent to the free surface, as depicted by the instantaneous streamlines. Over time, the Marangoni stress grows in magnitude and strengthens this reversal. The flow then separates from the free surface, giving rise to a vortex ring, as can be seen at $t=4.8$ in figure $8(\mathrm{a})$. The flow separation causes the blob-side pressure to drop (see figure 8(b)), or results in a "head-loss" as hypothesized by Hoepffner \& Paré (2013). Subsequently, fluid from the tip enters the boundary layer and flows toward the neck, filling it with fluid, as can be seen at $t=4.9$, thereby not only countering but negating the thinning effect of the bulk flow. As a result, the filament successfully escapes endpinching, and the neck begins to reopen by $t=5.0$.

To contrast the dynamics of the surfactant-covered filament that escapes endpinching and which has just been discussed from that of a surfactant-free filament that endpinches, figure 9 shows the time evolution of the pressure profile in the endpinching filament from figure 4a $\left(O h=10^{-3}, L_{0}=15, \Gamma_{0}=0\right)$. For the surfactant-free filament, in all the profiles leading up to pinch-off, the secondary pressure minimum in the blob (viz. $z_{\min }-z<0$ ) is noticeably absent, thereby demonstrating it to be a key physical signature of the escape process.

To provide more conclusive evidence that the escape process is indeed driven by Marangoni stress, figure 10(b) and (c) shows the results of simulations that have been carried out for the same filaments as in figures 6 and 7 respectively albeit with the Marangoni stress artificially turned off. This is achieved in the G/FEM formulation by switching-off the term $\mathbf{t} \cdot \nabla_{s} \sigma$ in equation (2.4). As a result, the surfactants are still distributed non-uniformly according to equation (2.7) and lead to differential lowering in surface tension, but do not produce a tangential stress on the interface. In accordance with our expectations, filaments with the Marangoni stress turned off do not escape endpinching. In summary, it has been demonstrated here in detail how surfactants, by generating Marangoni stress on the free surface, can retard and arrest endpinching of filaments even when the $O h$ (or filament viscosity) is small. 
a

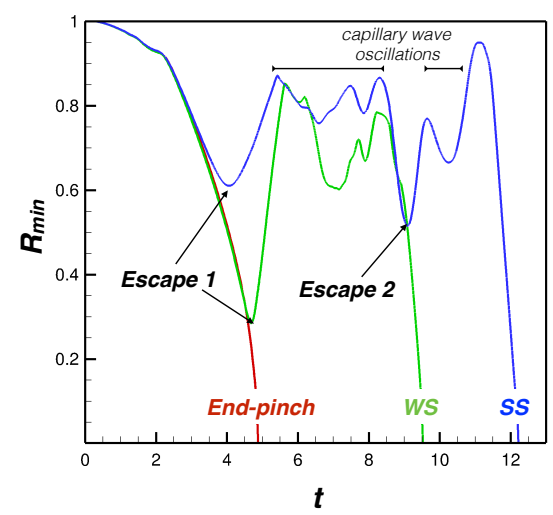

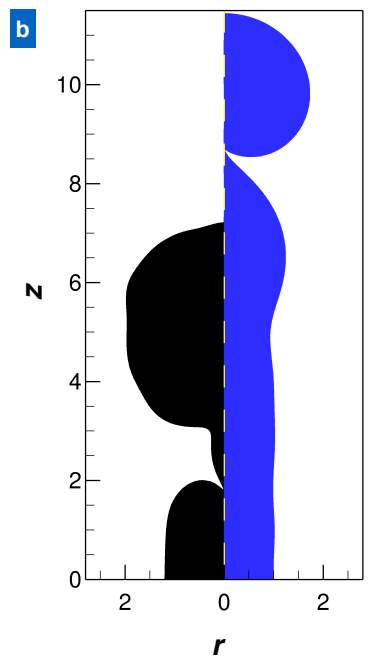

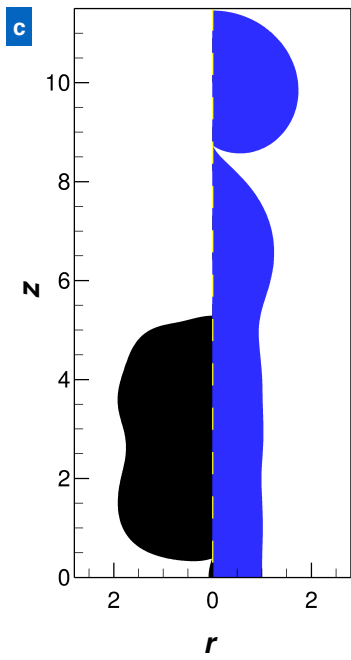

Figure 10. Demonstration that Marangoni stress is essential for escape from endpinching. (a) Computed variation of the minimum radius of the neck $R_{\min }$ with time $t$ for filaments of $O h=0.001$ and $L_{0}=15$. The filament without surfactant (red curve) endpinches. The surfactant-covered filaments (green and blue curves), both of which have $\Gamma_{0}=0.1$, escape from endpinching. The filament that is identified as WS (green curve), and for which $\beta=0.05$ and $P e=10$, escapes endpinching once. The filament that is identified as SS (blue curve), and for which $\beta=0.2$ and $P e=1,000$, escapes endpinching twice. The reopening of the neck, and escape from endpinching, shown here is a consequence of the presence of surfactants at the L-G interface. (b-c) Shapes of surfactant-covered filaments at breakup. Left-most panel: WS case. Right-most panel: SS case. In each panel, a side-by-side comparison is provided of the breakup shapes of filaments with the full model, i.e. with the Marangoni stress turned on (left side of the axis of symmetry, profile shaded black) and that with the Marangoni stress turned off (right side of the axis of symmetry, profile shaded blue). As can be seen from the filaments shaded in blue, artificially turning-off Marangoni stress for the two surfactant-covered filaments of part (a) leads to endpinching.

\section{Escape from Endpinching and Vorticity Generation at the Free Surface}

Escape from endpinching is well-known to occur also in surfactant-free filaments of intermediate Ohnesorge numbers $\left(O h \approx 10^{-2}\right)$, as first shown by Notz \& Basaran (2004). Simulations reveal that these filaments can undergo several-up to as many as ten - escape events during retraction, and that this process is independent of $L_{0}$ as long as the initial aspect ratio lies above the critical value $L_{0, c}$ required for pinch-off (Anthony et al. 2019). Interestingly, we observe several similarities between surfactantdriven escape from endpinching studied in the previous section and that in surfactant-free filaments (Hoepffner \& Paré 2013). A common feature of the dynamics in the two cases is the reversal of flow within a boundary layer which forms at the free surface. The boundary layer formation in surfactant-free filaments is driven by a viscous mechanism and therefore only occurs at higher values of $O h$ whereas it is driven by Marangoni stress in the presence of surfactants and can therefore also occur at low $O h$. In both cases, however, this reversal drives flow separation to take place which then results in the formation of a vortex ring in the blob, as can be clearly seen in figure 8 as well as in the experiments of Hoepffner \& Paré (2013).

The similarities in the two cases raise some important questions. First, why does a viscous boundary layer originate at the free surface at intermediate $O h$ in surfactant- 

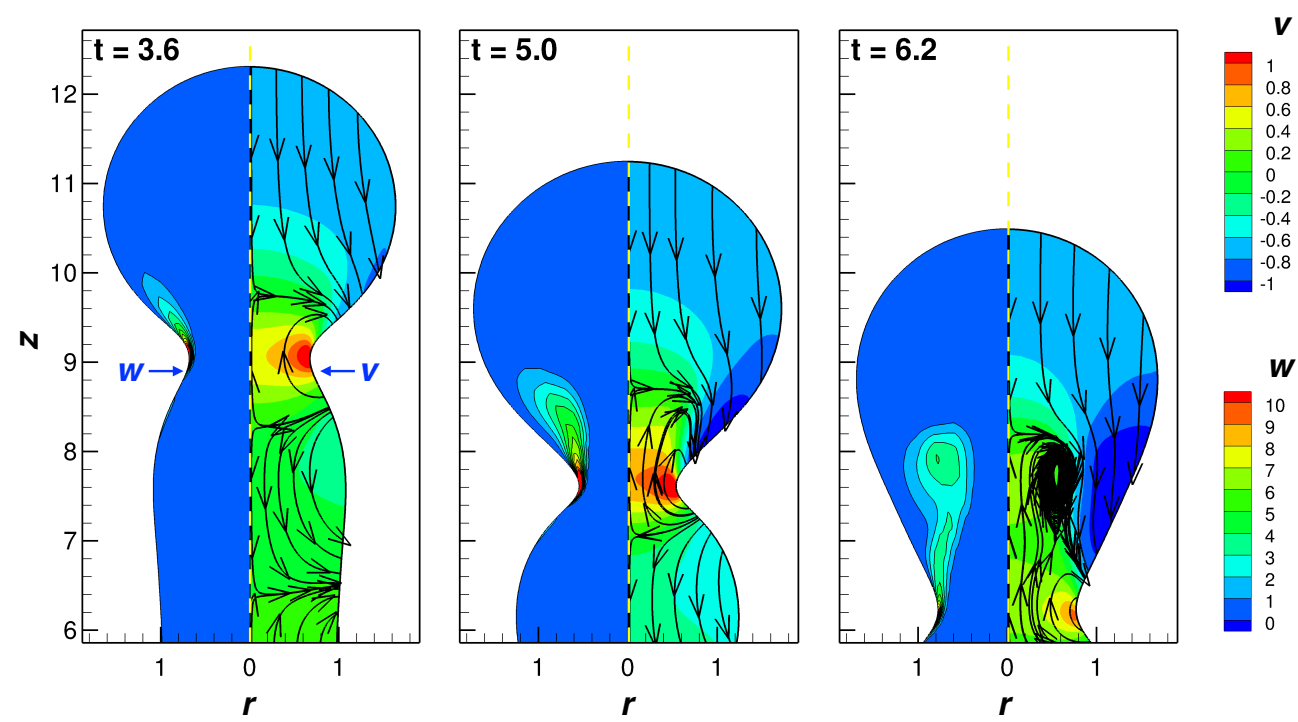

FiguRE 11. Vorticity and boundary layer formation in a surfactant-free filament of $O h=0.01$ and $L_{0}=15$ undergoing escape from endpinching. Snapshots of the dynamics at three different instants in time: at early times as blob formation is occurring (left), at an instant just prior to escape (middle), and at an instant after escape (right). In each panel, aside from the filament profile, contours of the axial velocity $v$ along with the instantaneous streamlines indicating the direction of flow are shown to the right of the axis of symmetry $r=0$ and contours of the norm of the vorticity $w=\|\mathbf{w}\|$ are shown to the left of $r=0$. Contour legends for each variable that apply to all three panels are shown on the extreme right.

free filaments? Second, how is it possible that two very distinct physical phenomenaMarangoni stresses at a filament's free surface and viscous effects in the filament's bulkcan cause the occurrence of the same set of complex physics that result in escape from endpinching? Specifically, it is natural to ask whether all escape events in filaments can be characterized as a single physical process. To answer these questions, it is useful to note that the formation of a boundary layer within a potential flow field goes hand-inhand with the generation of vorticity $\mathbf{w}=\nabla \times \mathbf{v}$ in that region (Deen 2012; Lighthill 1963). Therefore, it should be possible to appreciate the physical origins of boundary layer formation (and hence escape from endpinching), and to understand its dependence on system parameters, by studying the rate of change of vorticity in a contracting filament.

The introduction of vorticity into free surface flows via boundary layers has been extensively studied in the literature (Rood 1994; Lundgren \& Koumoutsakos 1999; Brøns et al. 2014). The starting point of such analyses is the microscopic vorticity transport equation. This well-known equation is obtained by taking the curl of the momentum equation (2.2), thereby eliminating the pressure gradient term, and can be written as

$$
\frac{D \mathbf{w}}{D t}=\mathbf{w} \cdot \nabla \mathbf{v}+O h \nabla^{2} \mathbf{w}
$$

The rate of generation of vorticity in a macroscopic volume $V(t)$ enclosed by the free surface $S(t)$ can then be obtained by integrating equation (7.1) over $V(t)$ and applying the appropriate kinematic (2.3) and traction (2.4) boundary conditions at the free surface. 
In a surfactant-free filament the macroscopic vorticity balance is

$$
\frac{D}{D t} \int_{V(t)} \mathbf{w} \mathrm{d} V=\int_{V(t)} \mathbf{w} \cdot \nabla \mathbf{v} \mathrm{d} V+2 O h \int_{S(t)} \mathbf{e}_{\theta} \mathbf{n} \cdot \nabla\left(\frac{\partial v_{n}}{\partial s}-\kappa_{t} v_{t}\right) \mathrm{d} S,
$$

where $\mathbf{e}_{\theta}$ is the unit vector pointing in the azimuthal direction and $\kappa_{t}$ is the principal curvature of the free surface in the plane of flow. The first term is the rate of accumulation of vorticity in $V(t)$. The second term describes the local amplification of vorticity produced by stretching of vortex lines. The last term on the right side of equation (7.2), a surface integral over the free surface, is the source term for vorticity. For an initially irrotational flow, vorticity is generated at the free surface and diffuses into the bulk from the L-G interface; the terms in the integral clearly show that the highest vorticity flux occurs in regions of high in-plane curvature where the tangential velocity is non-zero and the interface is undergoing motion in the direction normal to itself.

A perfect example of such a region is the thinning neck of a filament as it approaches endpinching. This is clearly evident in figure 11 which shows vorticity contours and streamlines for the filament of $O h=0.01$ and $L_{0}=15$ during the escape process. At early times (left frame), the flow around the highly-curved neck leads to the generation of small vorticity in its vicinity. As time proceeds, the curvature of the neck increases, and by $t=5.0$ (middle frame) a large amount of vorticity can be observed on the blob-side of the neck where the curvature, the rate of thinning of the neck, and the accompanying fluid motion in the normal direction to the interface, and that in the tangential direction to the free surface are all large. This vorticity generation coincides with the formation of the noticeable velocity boundary layer that is attached to the free, a substantive flow tangential to the free surface, and where flow separation will soon commence. These developments in the flow field signal that the escape process has thus begun: soon the back-flow within the boundary layer will refill the neck with fluid from the tip and cause it to reopen (Hoepffner \& Paré 2013). After the filament escapes endpinching, at $t=6.2$ (right frame), the viscous diffusion of the vorticity into the bulk leads to the growth of the boundary layer and the separation of the vortex ring.

The time-scale for vorticity generation $t_{w}$ can be obtained by balancing the first and last terms from equation (7.2), and is found to be the inertial-viscous time scale $t_{\mathbf{w}}=t_{c} / O h=\rho R^{2} / \mu$. When $O h$ is sufficiently small, $t_{\mathbf{w}}$ is significantly larger than $t_{c}$, the time scale over which inertio-capillary pinch-off takes place. Therefore when $O h \rightarrow 0$, significant vorticity generation cannot take place before pinch-off occurs in low-Oh filaments. This realization helps explain why these filaments cannot escape endpinching.

The discussion provided in the previous paragraphs can be extended to elucidate why low-Oh filaments can escape endpinching when they are covered with surfactant. In the presence of surfactants, the tangential stress boundary condition at the free surface (2.4) has an added term proportional to $\nabla_{s} \sigma$. Under this modification, the macroscopic vorticity balance becomes (see also $\mathrm{Xu}(2007)$ )

$$
\frac{D}{D t} \int_{V(t)} \mathbf{w} \mathrm{d} V=\int_{V(t)} \mathbf{w} \cdot \nabla \mathbf{v} \mathrm{d} V+2 O h \int_{S(t)} \mathbf{e}_{\theta} \mathbf{n} \cdot \nabla\left(\frac{\partial v_{n}}{\partial s}-\kappa_{t} v_{t}\right) \mathrm{d} S+\int_{S(t)} \mathbf{e}_{\theta} \mathbf{n} \cdot \nabla\left(-T_{n t}\right) \mathrm{d} S
$$

It is noteworthy that a new contribution to vorticity generation (that last surface integral on the right side of the equation) arising from the Marangoni stress at the free surface $T_{n t}=\mathbf{t} \cdot \nabla_{s} \sigma$ has now entered the vorticity balance. Moreover, it is important to note that this Marangoni-driven flux of vorticity from the free surface is independent of $O h$ 

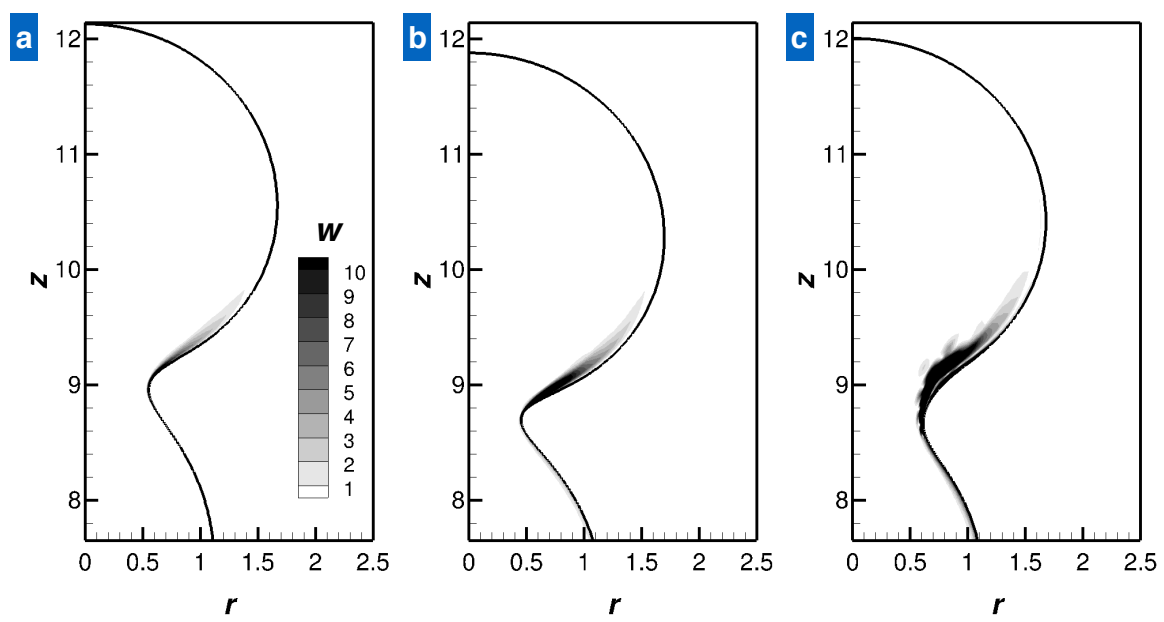

FiguRE 12. Vorticity in surfactant-covered filaments of $O h=0.001, L_{0}=15$, and $\Gamma_{0}=0.1$. Filament profiles and contours of the norm of the vorticity $w=\|\mathbf{w}\|$ for (a) a filament of $\beta=0.3$ and $P e=0.01$ at $t=3.9$, (b) a filament of $\beta=0.05$ and $P e=10$ at $t=4.2$, and (c) a filament of $\beta=0.2$ and $P e=1,000$ at $t=4.2$. The contour legend for $w$ shown in (a) applies in (a)-(c). The figure makes plain that large vorticity accumulation is a prerequisite for escape from endpinching (cases (b) and (c)).

and is therefore active even in nearly inviscid filaments, i.e. as $O h \rightarrow 0$. Furthermore, the vorticity flux due to the action of the Marangoni stress is large when the magnitude of $T_{n t}$ is large and its sign negative. Both of these conditions are always true on the blob-side in the vicinity of the neck in filaments undergoing breakup (cf. figure $7(\mathrm{c})$ ). A large amount of vorticity is generated in only those filaments that escape endpinching, as can be seen in simulation results shown in figure 12 .

For $O h \ll 1$, vorticity generation is wholly reliant on the last term in the macroscopic vorticity equation. Balancing the first and the last terms in the macroscopic vorticity equation shows that owing to the presence of surfactants, vorticity is now generated over a time scale equal to the capillary time scale: $t_{\mathbf{w}} \approx t_{c}$. As a result, velocity reversal and boundary layer formation can occur on the time-scale of pinch-off and surfactants can thereby induce escape from endpinching in filaments of low and intermediate values of $O h$. For larger values of $O h$, i.e. $O h \sim 1$ and $O h \gg 1$, a large amount of vorticity is still generated at the interface and transferred to the bulk but these effects are negated by the large viscous dissipation in the bulk, as has already been demonstrated in previous work (Notz \& Basaran 2004).

\section{Concluding Remarks and Future Work}

According to the foregoing results, escape from endpinching is driven by the same physical mechanism in both surfactant-free and surfactant-laden filaments. In both cases, the escape is due to large vorticity generation in the vicinity of the thinning neck of contracting filaments. Presence of surfactants at the L-G interface introduces an additional means due to the action of Marangoni stresses for filaments to escape from endpinching over and beyond what is possible in surfactant-free filaments. Hence, surfactant-covered filaments can escape from endpinching even in the limit of vanishingly small Ohnesorge number whereas surfactant-free filaments can only escape from endpinching when the Ohnesorge number is sufficiently large (Notz \& Basaran 2004; Hoepffner \& Paré 2013). 
Moreover, in any filament, if vorticity generation and accumulation can occur on the time-scale of pinch-off, then that filament can escape endpinching as it contracts. This is a noteworthy finding as spray formulations in industry are typically non-Newtonian in nature, and contain suspended particles or dissolved polymers and/or surface active compounds. The significance arises from the fact droplet size distributions in such sprays is governed to a large extent by the filament retraction and breakup process (Fezzaa \& Wang 2008; Villermaux 2007; Planchette et al. 2019). Therefore, one direct and important future application of the basic fluid mechanical understanding on vorticity generation and dissipation resulting from the present paper would be to provide important guidance on predicting the prevalence and significance of endpinching in a distribution of filaments formed in industrially relevant spray or atomization systems.

Filaments of sufficiently small aspect ratios do not break but can undergo oscillations and exhibit rich nonlinear dynamics (Notz \& Basaran 2004; Anthony et al. 2019). The oscillations of such free elongated drops (Lundgren \& Mansour 1988; Patzek et al. 1991; Basaran 1992; Apfel et al. 1997) as well as those of supported drops (Basaran \& De Paoli 1994; Wilkes \& Basaran 1997) and free/supported bubbles (Glazman 1984; Fyrillas \& Szeri 1995; Johnson \& Stebe 1994) are also of interest, e.g. in measurement of dynamic surface tension and other physical properties (Franses et al. 1996; Liao et al. 2006a). Current understanding of oscillations in such surfactant-laden systems is in its infancy compared to that of drops/bubbles of Newtonian fluids with clean interfaces, experimental investigations into surfactant effects are severely lacking in their appreciation of Marangoni stress effects. For example, in a recent study on drop breakup, Ponce-Torres et al. (2017) have carried out a theoretical analysis in which they have accounted for various effects due to the presence of surfactants at an interface. However, they have not accounted for the effects of Marangoni stresses when developing fits to data on frequencies of drop oscillations that they have obtained from experiments. Carrying out research aimed at overcoming these limitations constitutes a noteworthy and fruitful area of future study in fluid mechanics.

In addition to surface tension lowering and Marangoni stresses, surfactants also induce surface rheological effects when they deform against themselves at fluid interfaces. Surprisingly, little work has been done on problems involving interface rupture when surface viscosities are important. For example, Ponce-Torres et al. (2017) have uncovered that the increase in surfactant accumulation in satellite droplets during drop formation cannot be explained without accounting for surface viscosities and Martínez-Calvo \& Sevilla (2018) have shown that surface viscosities have a stabilizing influence on the dynamics in the Rayleigh-Plateau instability of liquid jets covered with a monolayer of insoluble surfactant. It would be of great fundamental and practical importance if analyses were carried out to study contraction of filaments in the presence of surface rheological effects by accounting for the effects of surface viscosities as in the aforementioned papers (PonceTorres et al. 2017; Martínez-Calvo \& Sevilla 2018).

A key aspect of the numerical analysis presented here is investigating whether Marangoni stress or the local lowering of surface tension-solutocapillary effect - is the dominant mechanism that determines whether escape from endpinching occurs during filament contraction. Historically, the approach taken to answer this question has been to use small values of the Peclet number $(P e \ll 1)$ or making the surfactant highly diffusive in order to eliminate surfactant concentration gradients to conclude whether Marangoni stress is weak or sub-dominant (Milliken et al. 1993; Craster et al. 2002) in a given free surface flow. However, that approach has the effect of simultaneously altering both the Marangoni and local solutocapillary effects, and is therefore not completely reliable. Here, we show the importance of Marangoni stress in escape by selectively turning-off 
Marangoni stress terms (cf. figure 10) in the traction boundary condition, a feat that is readily accomplished in a simulation but would be difficult if not impossible to realize in the laboratory. This approach was first used by Kamat et al. (2018) and it has allowed us here to make an unequivocal conclusion: Marangoni stress is the dominant mechanism in escape, as escape does not occur when Marangoni stresses are turned off but the solutocapillary effects continue to act without being impeded. Surfactant-laden free surface flows are typically complex and the different forces at play have co-dependencies that make it difficult to delineate the individual effect of each physical mechanism in isolation of others (Scriven \& Sternling 1960). According to the foregoing analyses, the technique employed here will prove useful in future investigations of free surface flows of surfactant-laden fluids. Indeed, this approach is likely to be especially useful in determining the importance of surface viscosities compared to the classical surface tension effects considered here, a debate that has not yet been conclusively settled.

In this paper and in virtually all others that have heretofore addressed the dynamics of contracting filaments, attention has been focused on the dynamics that occurs prior and up to the instant of first pinch-off (Notz \& Basaran 2004; Wang et al. 2019). The algorithm employed in this paper can be used to simulate the dynamics in the aftermath of the first pinch-off, as shown in Basaran et al. (2013). Among other things, it would be worthwhile to investigate how surfactants can modify the dynamics as a filament undergoes repeated pinch-offs as it recoils.

Declaration of Interests. The authors report no conflict of interest.

\section{REFERENCES}

Ambravaneswaran, B. \& Basaran, O. A. 1999 Effects of insoluble surfactants on the nonlinear deformation and breakup of stretching liquid bridges. Phys. Fluids 11 (5), 997.

Anthony, C. R., Kamat, P. M., Harris, M. T. \& Basaran, O. A. 2019 Dynamics of contracting filaments. Phys. Rev. Fluids 4 (9), 093601.

Apfel, R., Tian, Y., Jankovsky, J., Shi, T., Chen, X., Holt, R., Trinh, E., Croonquist, A., Thornton, K., Sacco, Jr., A., Coleman, C., Leslie, F. \& Matthiesen, D. 1997 Free Oscillations and Surfactant Studies of Superdeformed Drops in Microgravity. Phys. Rev. Lett. 78 (10), 1912-1915.

Basaran, O. A. 1992 Nonlinear oscillations of viscous liquid drops. J. Fluid Mech. 241, 169198.

Basaran, O. A. 2002 Small-scale free surface flows with breakup: Drop formation and emerging applications. AIChE Journal 48 (9), 1842-1848.

Basaran, O. A. \& De Paoli, D.W. 1994 Nonlinear oscillations of pendant drops. Phys. Fluids $6(9), 2923$.

Basaran, O. A., GaO, H. \& Bhat, P. P. 2013 Nonstandard inkjets. Annual Review of Fluid Mechanics 45, 85-113.

Brøns, M., Thompson, M. C., Leweke, T. \& Hourigan, K. 2014 Vorticity generation and conservation for two-dimensional interfaces and boundaries. J. Fluid Mech. 758, 63-93.

Castrejón-Pita, A. A., Castrejón-Pita, J. R. \& Hutchings, I. M. 2012 Breakup of Liquid Filaments. Phys. Rev. Lett. 108 (7), 074506.

Castrejón-Pita, J. R., Baxter, W. R. S., Morgan, J., Temple, S., Martin, G. D. \& Hutchings, IAN M. 2013 Future, opportunities and challenges of inkjet technologies. Atomization and Sprays 23 (6), 541-565.

Chen, Y. J. \& Steen, P. H. 1997 Dynamics of inviscid capillary breakup: collapse and pinchoff of a film bridge. J. Fluid Mech. 341, 245-267.

Christodoulou, K.N. \& Scriven, L. E. 1992 Discretization of free surface flows and other moving boundary problems. J. Comput. Phys. 99 (1), 39-55. 
Craster, R. V., Matar, O. K. \& Papageorgiou, D. T. 2002 Pinchoff and satellite formation in surfactant covered viscous threads. Phys. Fluids 14 (4), 1364.

Culick, F. E. C. 1960 Comments on a ruptured soap film. J. Appl. Phys. 31 (6), 1128-1129.

Day, R. F., Hinch, E. J. \& Lister, J. R. 1998 Self-Similar Capillary Pinchoff of an Inviscid Fluid. Physical Review Letters 80 (4), 704-707.

Deen, W. M. 2012 Analysis of Transport Phenomena, 2nd edn. New York: Oxford University Press.

Derby, B. 2010 Inkjet printing of functional and structural materials: fluid property requirements, feature stability, and resolution. Ann. Rev. Mat. Res. 40, 395-414.

Driessen, T., Jeurissen, R., Wijshoff, H., Toschi, F. \& Lohse, D. 2013 Stability of viscous long liquid filaments. Phys. Fluids 25 (6), 1-8.

Eggers, J. 2005 Drop formation - an overview. Zeitschrift für Angew. Math. und Mech. 85 (6), $400-410$.

Eggers, J. \& Dupont, T. F. 1994 Drop formation in a one-dimensional approximation of the NavierStokes equation. J. Fluid Mech. 262, 205-211.

Eggers, J. \& Villermaux, E. 2008 Physics of liquid jets. Reports Prog. Phys. 71 (0001), 1-79.

FEZZAA, K. \& WANG, Y. 2008 Ultrafast x-ray phase-contrast imaging of the initial coalescence phase of two water droplets. Phys. Rev. Lett. 100 (10), 104501.

Franses, E. I., Basaran, O. A. \& Chang, C.-H. 1996 Techniques to measure dynamic surface tension. Curr. Op. Coll. \& Int. Sci. 1 (2), 296-303.

Fyrillas, M. M. \& Szeri, A. J. 1995 Dissolution or growth of soluble spherical oscillating bubbles: the effect of surfactants. J. Fluid Mech. 289, 295.

Glazman, R. E. 1984 Damping of bubble oscillations induced by transport of surfactants between the adsorbed film and the bulk solution. J. Acoust. Soc. Am. 76 (3), 890-896.

Gresho, P. M., Lee, R. L. \& Sani, R. C. 1979 On the time-dependent solution of the incompressible Navier-Stokes equations in two and three dimensions. In Recent Adv. Numer. Methods Fluids, Vol. 1 (ed. C. Taylor \& K. Morgan), pp. 27-79. Swansea: Pineridge.

Hoepffner, J. \& Paré, G. 2013 Recoil of a liquid filament: escape from pinch-off through creation of a vortex ring. J. Fluid Mech. 734, 183-197.

Johnson, D. O. \& Stebe, K. J. 1994 Oscillating Bubble Tensiometry: A Method for Measuring the Surfactant Adsorptive-Desorptive Kinetics and the Surface Dilatational Viscosity. J. Colloid Interface Sci. 168 (1), 21-31.

Kamat, P. M., Castrejón-Pita, A. A., Castrejón-Pita, J. R., Wagoner, B. W., Anthony, C. R. \& A., Basaran O. 2020 Experimental and computational study of surfactant-driven escape from endpinching during filament retraction. to be submitted to: Phys. Rev. Fluids .

Kamat, P. M., Wagoner, B. W., Thete, S. S. \& Basaran, O. A. 2018 Role of marangoni stress during breakup of surfactant-covered liquid threads: Reduced rates of thinning and microthread cascades. Physical Review Fluids 3 (4), 043602.

LiaO, Y.-C., Basaran, O. A. \& Franses, E. I. $2006 a$ Effects of dynamic surface tension and fluid flow on the oscillations of a supported bubble. Coll. and Surf. A: Phys. Eng. Asp. 282, 183-202.

Liao, Y.-C., Franses, E. I. \& Basaran, O. A. $2006 b$ Deformation and breakup of a stretching liquid bridge covered with an insoluble surfactant monolayer. Phys. Fluids 18 (2), 022101.

Lighthill, M. J. 1963 Laminar boundary layers.. Oxford University Press, USA.

Lundgren, T. \& Koumoutsakos, P. 1999 On the generation of vorticity at a free surface. $J$. Fluid Mech. 382, 351-366.

Lundgren, T. S. \& Mansour, N. N. 1988 Oscillations of drops in zero gravity with weak viscous effects. J. of Fluid Mech. 194, 479-510.

Martínez-Calvo, A., Rivero-Rodríguez, J., Scheid, B. \& Sevilla, A. 2020 Natural breakup and satellite formation regimes of surfactant-laden liquid threads. Journal of Fluid Mechanics 883, A35.

Martínez-Calvo, A. \& Sevilla, A. 2018 Temporal stability of free liquid threads with surface viscoelasticity. Journal of Fluid Mechanics 846, 877-901.

McGough, P. T. \& Basaran, O. A. 2006 Repeated Formation of Fluid Threads in Breakup of a Surfactant-Covered Jet. Phys. Rev. Lett. 96 (5), 054502. 
Milliken, W. J., Stone, H. A. \& Leal, L. G. 1993 The effect of surfactant on the transient motion of Newtonian drops. Phys. Fluids A Fluid Dyn. 5 (1), 69.

Notz, P. K. \& Basaran, O. A. 2004 Dynamics and breakup of a contracting liquid filament. J. Fluid Mech. 512, 223-256.

Notz, P. K., Chen, A. U. \& Basaran, O. A. 2001 Satellite drops: Unexpected dynamics and change of scaling during pinch-off. Physics of Fluids 13 (3), 549-551.

Patzek, T. W., Benner, R. E., Basaran, O. A. \& Scriven, L. E. 1991 Nonlinear oscillations of inviscid free drops. J. of Comp. Phys. 97 (2), 489-515.

Planchette, C., Marangon, F., Hsiao, W.-K. \& Brenn, G. 2019 Breakup of asymmetric liquid ligaments. Phys. Rev. Fluids 4 (12), 124004.

Ponce-Torres, A., Montanero, J. M., Herrada, M. A., Vega, E. J. \& Vega, J. M. 2017 Influence of the Surface Viscosity on the Breakup of a Surfactant-Laden Drop. Phys. Rev. Lett. 118 (2), 1-5.

Roché, M., Aytouna, M., Bonn, D. \& Kellay, H. 2009 Effect of Surface Tension Variations on the Pinch-Off Behavior of Small Fluid Drops in the Presence of Surfactants. Physical Review Letters 103 (26), 264501.

Rood, E. P. 1994 Interpreting Vortex Interactions With a Free Surface. J. Fluids Eng. 116, 91-94.

Rosen, M. J. 2004 Surfactants and interfacial phenomena, 3rd edn. John Wiley \& Sons, Inc.

Schulkes, R.M.S.M. 1996 The Contraction of liquid filaments. J. Fluid Mech. 309, 277-300.

Scriven, L. E. \& Sternling, C. V. 1960 The Marangoni effects. Nature 187 (4733), 186-188.

Stone, H. A. 1990 A simple derivation of the time-dependent convective-diffusion equation for surfactant transport along a deforming interface. Phys. Fluids A Fluid Dyn. 2 (1), 111.

Stone, H. A., Bentley, B.J. \& Leal, L. G. 1986 An experimental study of transient effects in the breakup of viscous drops. J. Fluid Mech. 173, 131-158.

Stone, H. A. \& Leal, L. G. $1989 a$ Relaxation and breakup of an initially extended drop in an otherwise quiescent fluid. J. Fluid Mech. 198, 399.

Stone, H. A. \& Leal, L. G. $1989 b$ The influence of initial deformation on drop breakup in subcritical time-dependent flows at low Reynolds numbers. J. Fluid Mech. 206, 223.

Strang, G. \& Fix, G. 1973 An Analysis of the Finite Element Method. Wellesley-Cambridge.

TAYlor, G. I. 1959 The Dynamics of Thin Theets of Fluid. III. Disintegration of Fluid Sheets. Proc. R. Soc. A Math. Phys. Eng. Sci. 253 (1274), 313-321.

Timmermans, M.-L. E. \& Lister, J. R. 2002 The effect of surfactant on the stability of a liquid thread. J. Fluid Mech. 459, 289-306.

Villermaux, E. 2007 Fragmentation. Annu. Rev. Fluid Mech. 39 (1), 419-446.

Wang, F, Contò, F. P., Naz, N., Castrejón-Pita, J. R., Castrejón-Pita, A. A., Bailey, C. G., WAng, W., Feng, J. J. \& Sui, Y. 2019 A fate-alternating transitional regime in contracting liquid filaments. J. Fluid Mech. 860, 640-653.

Wilkes, E. D. \& Basaran, O. A. 1997 Forced oscillations of pendant (sessile) drops. Phys. Fluids 9 (6), 1512-1528.

Xu, Q. 2007 COMPUTATIONAL AND THEORETICAL ANALYSIS OF INK-JETS DROP FORMATION AND BREAKUP. PhD thesis, Purdue University.

Yeo, Y., Chen, A. U., Basaran, O. A. \& Park, K. 2004 Solvent exchange method: a novel microencapsulation technique using dual microdispensers. Pharm. Res. 21 (8), 1419-1427.

Zhang, X., Harris, M. T. \& Basaran, O. A. 1994 Measurement of dynamic surface tension by a growing drop technique. J. Coll. Int. Sci. 168 (1), 47-60. 